\title{
Changes in Attitudes toward Nutrition after an Education Program in Polish Teenagers: Report from the ABC of Healthy Eating Study
}

\section{Abstract}

The sustainability of education focused on improving teenagers' attitudes toward nutrition from a longer-term perspective has not yet been studied extensively. The aim of this study was to determine the sustainability of attitudes toward nutrition after 3 and 9 months among Polish teenagers in a follow-up study. An education-based intervention study was carried out among 464 Polish teenagers aged 11-12 years (educated/control group: 319/145). In the educated group, a multicomponent, school-based education program lasting three weeks and covering five diet-related and lifestyle-related topics was implemented. Attitudes toward nutrition were determined with a shorter version (TFEQ10; 10 statements) of a three-factor eating questionnaire (TFEQ13) dedicated to school-aged adolescents, where three subscales were developed: Emotional Eating (EE: 2 statements, 0-6 points), Uncontrolled Eating (UE: 5 statements, $0-15$ points), and Cognitive Restraint of Eating (CRE: 3 statements, $0-9$ points). The data was collected 3 times: (i) before education (at baseline), (ii) after 3 months to measure the short-term effect of education, (iii) after 9 months to measure the mediumterm effect of education. Differences between groups (educated vs. control or baseline vs. follow-up) were verified with the MannWhitney test. At the baseline, the mean values for all three scales were not significantly different between educated and control groups, calculated separately for boys and girls as well as for normal or overweight/obese teenagers, with the exception for Emotional Eating in the total sample and normal weight students. After 3-month follow-up, the mean values decreased in an educated group for all three subscales, for the total sample (EE: 1.3 vs 1.2 ; UE: 5.2 vs 4.7; CRE: 4.2 vs 4.0), for both sexes, and both body weight subpopulations, while increased or remained on the same level in control group (for total sample EE: 1.5 vs 1.6; UE: 5.6 vs 5.8; CRE: 4.1 vs 4.2). After 9 months, in the educated group, the means slightly increased when compared to the means after 3 months, but were still lower than those at the baseline. This study has shown that nutritional education of teenagers 11-12 years gives a long-lasting effect of attitudes toward nutrition (Emotional Eating and Uncontrolled Eating) visible after 3 months, and to a less extent after 9 months. We suppose, that improvement of the attitudes toward nutrition in teenagers, education programs should be regularly conducted and addressed not only to children but also to the adults responsible for children's nutrition.

\section{Conflict of Interest}

There is no conflict of interest. The study was financially supported by Carrefour Foundation (Agreement ABC No. 1/2014; Agreement ABC No. 2/2016) and each scientific center from sources of the Polish Ministry of Sciences and Higher Education 